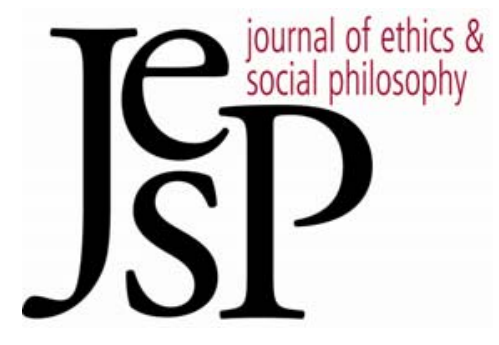

\title{
ESSENTIALLY COMPARATIVE CONCEPTS
}

BY JONATHAN DANCY

Journal OF ETHICs \& Social PhILOSOPhy

VOL. 1, NO. 2 | JUNE 2005

URL: WWW.JESP.ORG 


\title{
Essentially Comparative Concepts
}

\author{
Jonathan Dancy
}

\begin{abstract}
T N HIS "RETHINKING THE Good"1, Larry Temkin discusses the claim that equality is essentially comparative (EEC). The notion of the essentially comparative is characterized roughly as follows:

A principle (or moral idea) $f$, is essentially comparative if the relevant and significant factors for comparing two alternatives regarding $f$ may vary depending on the alternatives being compared; and, more specifically, $f$ is essentially pairwise comparative if one must directly compare two alternatives in order to determine their relative ranking regarding $f$. (p. 304)
\end{abstract}

Temkin writes, in a section which concerns Parfit's Mere Addition Paradox:

\begin{abstract}
The advocate of EEC believes inequality is not objectionable when it is brought about by the mere addition of extra people all of whom have lives worth living and who affect no one else, and where the alternative is a situation not where those people are better off, but where they don't exist. (p. 304)
\end{abstract}

There is something odd about this. There seem to be two views being squashed into one. The first view is that inequality is not objectionable when it is caused or reached in a certain way; I am going to call this the "provenance view." The second is that a situation which contains inequality may be objectionable for that reason when compared with some alternatives and not when compared with others. I will call this "alternative complementarity." The former is not a remark about alternatives, but about the way in which the causal (and other?) antecedents of a situation can affect the question whether it is the worse for the presence of a certain feature. Both of these claims are holistic, and they may in one way or another go together, but they are not identical. They are made to seem closer than they are by starting from an example where situation $B$ is reached from situation $\mathrm{A}$, and the two alternatives are $\mathrm{A}$ and $\mathrm{B}$. But this is a special case. The claim that equality is essentially comparative should say nothing officially about the question whether the causal (and other?) antecedents of a situation can affect whether it is the worse or not for the presence of a certain feature. Thoughts of this sort should only come in when considerations of causal antecedents or routes are involved in the construction of the relevant alternative - as they do with the Mere Addition Paradox.

\footnotetext{
${ }^{1}$ Larry Temkin "Rethinking the Good. Moral Ideals and the Nature of Practical Reasoning” in J. Dancy, ed., Reading Parfit (Oxford: Blackwell, 1997), pp. 290-345. All otherwise unattributed references will be to this paper.
} 
Temkin makes two comments on EEC. First, he says that if it is true "there is no fact of the matter as to how good a situation really is regarding $f$, considered just by itself. How good or bad it is depends on the alternative with which it is compared." (ibid.) Second, he says that "there may be no fact of the matter as to how two situations compare considered 'purely' abstractly. One may need to know who their 'members' are or how they've come about." (ibid.) But the idea that this second comment goes with the first one in such a way that they are part and parcel of the same essentially comparative conception of inequality (in the light of which he says that if EEC is true, considerations of equality are going to be sensitive to considerations about identity) is a consequence of the illegitimate way in which he has earlier run together thoughts about alternatives and thoughts about provenance, or causal origins. It is not officially part of any thought about the comparative at all.

The next point is that Temkin takes himself to be expounding Parfit on these matters. But Parfit's view, at least at one crucial place, seems not to be as represented by Temkin. Parfit is discussing the Mere Addition Paradox.

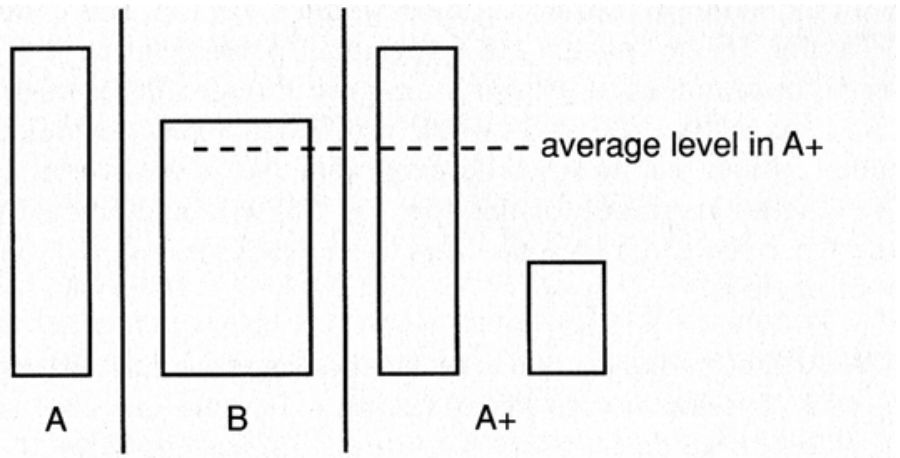

Fig. 1 The Mere Addition Paradox

In this paradox, A and B are two worlds. A contains fewer people than B does, but the quality of life of those in $A$ is higher than that of those in $B$. $\mathrm{A}+$ is reached by adding to A a separate world in which the average quality of life is much lower than that of those in A, though the lives concerned are still worth living. The worry is that $A$ is better than $B$ (because the quality of life in $A$ in higher), and $B$ is better than $A+$ for the same reason, but $A+$ is not worse than $A$, because it has all the value that $A$ has plus whatever value the less worthwhile lives that we have added may have. This is a paradox because it breaches the transitivity of value.

When talking about equality, Parfit says: "It was claimed above that the inequality of $\mathrm{A}+$ is a bad feature. I accept this claim." 2 If this were all that Parfit has to say on the matter, we should conclude that Temkin has significantly misrepresented him (or perhaps silently corrected an obvious error). For he reports Parfit as holding that inequality is not objectionable

2 Reasons and Persons (Oxford: Clarendon Press, 1984), p. 425. 
when brought about in certain ways (e.g. by moving from A to A+). And Parfit explicitly says, of just such a case, that its inequality is a bad feature. But to be fair, Parfit also says that "when inequality is, as here, produced by Mere Addition, it does not make the outcome worse ... this inequality does not make A+ worse than A."3 Presuming that Parfit has not simply contradicted himself, how is he to be interpreted? There are two ways of reading what Parfit says, both alluded to in the sentence I have just quoted.

The first works with a contrast between absolute and comparative value. The idea here is that that $\mathrm{A}+$ is the worse for its inequality, and, in terms of equality, much worse than $\mathrm{A}$ (which is perfectly equal), but that in assessing the relative merits of $\mathrm{A}$ and $\mathrm{A}+$ the inequality of $\mathrm{A}+$ is irrelevant. $A+$ may be worse than $A$, but even if so the fact that it is worse in terms of equality does not contribute to this result. I will call this reading the "bracketing view," because it involves bracketing some aspects of the values of two possibilities when we try to decide which is to be preferred.

A quite different way of understanding Parfit appeals to Moore's doctrine of organic wholes, and I will call this the "Moorean view." Moore took it that an organic whole is a whole in which each part retains its "intrinsic" value, but which may be made the better or the worse by the presence of that part in ways that are not explained by, and may be quite different in style from, that intrinsic value. For instance, the pain inflicted in punishment is as bad as it would be elsewhere, but the whole [crime and punishment] is less bad than the crime would be alone. ${ }^{4}$

There is a significant difference between these two interpretations. One appeals to thoughts about comparison and alternatives; the other does not.

Let us suppose that both these positions, the bracketing view and the Moorean view, are coherent. In assessing them, we should consider what further alternatives there are. (This is not just a topical joke; it is true on any account.) We have already seen two more. The provenance view is in a position to hold that because of how $\mathrm{A}+$ was reached its inequality is not a feature in virtue of which it is worse; the inequality is not bad here at all. This approach appears to sit better with, and even to explain, the idea that the inequality involved in $\mathrm{A}+$ does not affect the comparative merits of A and A+. However, as I argued above, thoughts about origin are not proper parts of EEC. The final possibility, then, is alternative complementarity - the claim that the contribution of any feature of one alternative to its overall value may be affected by the nature of its alternative(s). If we add to this the claim that a feature cannot contribute a value

3 ibid. Note that Parfit could certainly argue that the inequality of $\mathrm{A}+$ is not a reason for failing to bring about $\mathrm{A}+$ if we are starting from $\mathrm{A}-$ but it would be if we were starting from B.

4 I discuss this view, and compare it with the view which I favor, in my "Was Moore right about Punishment?," in Themes from G. E. Moore: New Essays in Epistemology and Ethics, eds. S Nuccetelli and G. Seay (Oxford: Oxford University Press, forthcoming). 
it has not got, we get a different account of why the inequality is not bad here; it is not bad because the alternative to A+ is A.

Of course, one can hold the provenance view together with alternative complementarity. They are only incompatible in the ways in which they would explain why the inequality of $\mathrm{A}+$ is not had there - if it is not. Further, there is a perspective from which the combination of the two is rather attractive. For we can see both positions as expressions of a certain holistic account of value; the first looks to an ancient doctrine of organic unities or wholes (not Moore's), and maintains that the value of a given feature may vary from one context to another. ${ }^{5}$ Thus the inequality is not bad here though it would be bad elsewhere. The second sees the various alternatives in a choice situation as in a relevant sense parts of a whole, subject again to organicity. The value of one alternative can be affected by the nature of the choice situation, i.e. by the nature of other alternatives. And in line with this, since the value of the parts is a function of the value of the whole (and vice versa), the value of the inequality of $\mathrm{A}+$ is affected by the fact that the alternative to A+ is A.

Is alternative complementarity the position which Temkin ends up recommending - the view that the main contributing concepts, as well as the concept of all-things-considered better than, are essentially comparative? It looks as if the answer is yes, if I am right in claiming that alternative complementarity constitutes the proper core of EEC. So there are four positions. Parfit holds that the inequality is bad but does not make this outcome worse than that one, and we have seen two ways of running this idea: the bracketing view and the Moorean view. The other two hold that the inequality is not here bad, but give different explanations of the fact. Those explanations only collapse into each other in the special case where an origin is an alternative, as in the Mere Addition Paradox.

The reason why it is so important to distinguish alternative complementarity from any thought about origins and routes is that the latter might just allow us to retain the claim that all-things-considered better than is transitive. So we should consider which of our four positions do in fact have that effect.

The bracketing view has the peculiar consequence that if one considers the value of the different options separately, one will get results which necessarily retain transitivity. But if one compares the different options, one will not. The oddity of this result may be thought likely to render this first position unstable.

\footnotetext{
${ }^{5}$ For a discussion of the difference between this doctrine and Moore's, see my Ethics Without Principles (Oxford: Clarendon Press, 2004), ch. 9. I should say that, even if we recognize Parfit's acceptance of the view that the inequality of $\mathrm{A}+$ is itself bad, we could see this as stemming not from a conception of intrinsic value, as I suggested above, but just from the sense that despite its origin it is still bad (though it might have been not bad, as it were). If we take this line, we see Parfit as adopting the bracketing view, since the actual value he attributes to the inequality of $\mathrm{A}+$ is incidental to the main point.
} 
The Moorean view, which appeals to the notion of an organic whole, retains transitivity. To establish this, we need to remember the details of Moore's view, and in particular his distinction between "good on the whole" and "good-as-a-whole." The question whether a complex is "good on the whole" is the question whether the value of the whole plus the intrinsic value of the parts is positive or negative. The question whether something is "good as a whole" is the question whether "there arises from the combination [of two evils] a positive good which is greater than the difference between the sum of the two evils and the demerit of either singly." 6

To illustrate: with this distinction, Moore can say that a complex is "good as a whole" partly because of the presence of an evil, even though the presence of that evil is sufficient to prevent the complex from being "good on the whole." If the complex [crime + punishment] is such a case, it is no doubt not good on the whole, since it is something the world would have been better without. But it may possibly still be good as a whole, and would be if, as he puts it, there arises from the combination a positive good which is greater than the difference between the sum of the two evils and the demerit of either singly. So if the crime is -5 and the pain inflicted is also -5 , but the combination creates a good of +6 , then the complex [crime + punishment] is good as a whole, but not good on the whole. On the whole it has the value of -4 .

This position retains transitivity, whichever sort of value one is comparing. These values can all be expressed numerically, and they are not affected by the alternatives with which we compare them.

We should then expect that thoughts about origins and routes will do nothing to undermine transitivity. Alternative complementarity, of course, destroys transitivity. But the other approach, our third, which allows that the inequality of $\mathrm{A}+$ is not bad if caused in a certain way, does not accept that the value of the whole is subject to change according to the alternatives with which it is compared. This surely amounts to the retention of transitivity. It therefore throws us back to the Mere Addition Paradox, and the consequence that if we accept transitivity we are involved in a contradiction. For the role of the attempt to show that $\mathrm{A}+$ is not the worse for the presence of inequality is to beat off the attempt to respond to the paradox by arguing that $\mathrm{A}+$ is worse than $\mathrm{A}$ because of its inequality. If we had $\mathrm{A}>\mathrm{A}+, \mathrm{A}>\mathrm{B}, \mathrm{B}>\mathrm{A}+$, the paradox would be resolved.

This seems to mean that, of all the four positions, the only one that escapes real incoherence is EEC. The only incoherence it introduces is the incoherence of intransitivity. (Here I am moving back towards agreement with Temkin.)

${ }^{6}$ Principia Ethica (Cambridge: Cambridge University Press, 1903), p. 215. 
I now turn to ask whether Temkin's failure to distinguish thoughts about origins and routes from thoughts about alternatives might not have further, more damaging consequences. He suggests that "the best way to avoid intransitivity in our all-things-considered judgements will be ... to deny that any essentially comparative principles or ideals are relevant to our all-things-considered judgements.” (p. 308) He suggests that such a position involves:

\begin{abstract}
an intrinsic aspect view (IA): where, roughly, how good or bad a situation is regarding some principle, $f$, will be an intrinsic feature of that situation. That is, it will not depend on the alternative that situation is compared with, but solely on features internal to the situation. (p. 309)
\end{abstract}

Temkin comments, "On IA, how a situation has come about, or who its members are, will be irrelevant to the abstract impersonal judgement about how it fares regarding f." He is, I recognize, uneasy about this, because he concedes that there is a perfectly good sense in which the identity of the protagonists is an intrinsic feature of the situation, and can be relevant to our overall judgements in various ways. He writes in a footnote:

\footnotetext{
I am not denying the obvious fact that there is a straightforward sense in which people's identities can be regarded as intrinsic, or internal, features of situations. But on IA people's identities cannot be relevant to how good or bad a situation is regarding such ideals as equality, utility, maximin or perfectionism - at least not if IA is to avoid the intransitivities arising from essentially comparative views. Moreover, though I did not argue this, I take the whole point of IA is to deny that identity per se is (or could be) relevant to our abstract, impartial, static judgements about how good situations themselves are. (fn. 44, p. 344)
}

What is causing the problem is an equivocation on the notion of the "internal" or "intrinsic." "Intrinsic" is what we used to call a trouserword, meaning thereby that it gets its sense from whatever it is here contrasted with. There are two quite distinct contrasts at issue. The first is between the nature of this alternative and that of others. The second is between the nature of this situation, viewed in a sort of freeze-frame, and its broader nature set in its historical context. Crucially, one could easily deny the sense of freeze-frame evaluations, on the ground that they abstract from blatantly relevant features, but it is much harder to make this move with respect to the attempt to evaluate one alternative by itself. Now the supposed need not to consider who people are (identities) derives from the freeze-frame approach, because identity cannot matter at a timeslice. But once we recognize that the objects we are considering (worlds, actions or whatever) have histories that are relevant to the ways in which value derives from their various features, the need to abstract from the 
identities of the protagonists vanishes (quite apart from the possibility of doing so). It is surely clear that it is relevant to the value of a situation whether, for instance, the person who is in possession of this good has a right to it, and such matters are sensitive to history, i.e. to the question whether the same person achieved such and such in the past.

Having made this move, however, we can continue to deny alternative complementarity for the diachronic objects we are evaluating. That is what IA should be taken to deny. It is not officially interested in identity at all. And this shows that Parfit himself could accept IA, if his real position is the Moorean or the provenance view

So Temkin's comment involves just the mistake I am here trying, yet again, to point out. Whenever Temkin uses the word "abstract," we know that he is talking about the supposed need, according to EEC, to consider merely the abstract nature of a situation, without taking into account its origin or the route by which it was reached. And we also know that the supposed need to do this has nothing directly to do with thoughts about IA and essentially comparative concepts. There is a danger, therefore, that Temkin will be drawn into comparisons and arguments that depend on this slide. And this is just what we see.

He first argues that if we adopted IA throughout, we could avoid the Mere Addition Paradox, because Parfit needed to appeal to EEC in order to defend the Paradox. I have no quarrel with this part of his argument. And if he is right here, a very attractive option opens up for us - one which is even available to Parfit, if the real thrust of his remarks about inequality concerns origins and routes rather than comparison and alternatives. We adopt IA in general, and thus retain transitivity, while "solving" the Mere Addition Paradox in other ways.

But Temkin goes on to argue that the wholesale adoption of IA is more or less impossible, because most of the main "principles or ideals" are essentially comparative, i.e. IA does not hold for them. So he floats this option before us, and then whisks it away. As it turns out, however, most of his arguments involve showing that how a situation has come about, or who its members are, rightly affect our judgement about how it fares regarding those ideals. And all such arguments will miss the point, according to me. Thoughts about origins, routes and identity are not at odds with IA.

This is an important matter, and bears detailed investigation. Temkin first considers a principle of justice: "maximin: the best outcome is the one in which the worst-off people are best off." (p. 316)

He argues that maximin is essentially comparative. But his argument is effectively that M-type considerations cannot be captured in freezeframes, and so are contraventions of IA, and so involve the essentially comparative. And we have seen that this involves a fallacy. The fact that we cannot capture the relevance of a feature in freeze-frames does nothing to show that it is essentially comparative. For instance, Temkin asks, of two worlds I and II, "suppose II resulted from I via groups B, C and $\mathrm{D}$ dying off from old age, and the lives of the A-group being adversely affected." Parfit contends it would be absurd to say II was better than I, even regarding maximin. He writes "... Maximin reflects our special concern for 
those worst-off, and this concern is not alleviated by the prospect of the worst-off dying. ... Maximin ... is not - and cannot be - fully reflected in a situation's abstract, internal features" (pp. 317-18). In another case (p. 318) he suggests that our relative assessment of worlds VI and VII with respect to maximin will be affected by the question "if VII and VI had entirely different populations, or if VII had resulted from VI by redistribution." This last is again a thought not about alternatives, but about origins, routes and identity.

Slightly different considerations arise when we turn to arguments concerning: "the person-affecting view (PAV): where, roughly, how good or bad an outcome is, is a function of the extent to which it affects people for better or worse." (p. 319)

Here we should bear in mind at the outset that if we are concerned about how our choices affect people, it is going to be impossible to avoid questions about who is who. ${ }^{7}$ We have to know whether the person after the change is the same as the one before it in order to know if anyone has been benefited. So it is not surprising that Temkin announces that PAV introduces considerations that are essentially comparative. Our response to this announcement should, again, be cautious. For we have decided that IA does not rule out thoughts about identity in the way that Temkin supposes. So it might yet be that PAV introduces considerations which require thoughts about identity, but which are not for that reason essentially comparative.

Temkin's arguments are all to the effect that since it introduces identity, PAV is comparative. For instance, in arguing for the conclusion that "PAV is essentially pairwise comparative. Its content cannot be captured by an intrinsic aspect view," he writes. "Clearly then, on PAV our judgement about how A and B compare will depend crucially on the identities of the people involved" (p. 320). However he goes on to say "Correspondingly, A's and B's desirability will not depend, in the relevant sense required by IA, solely on their internal features, but on both the alternatives they represent and the ones with which they are compared." This argument appears more to the point. If Temkin can establish, via thoughts about identity, that PAV introduces considerations that depend on alternatives in the required sense, he does have an argument that IA cannot capture PAV.

In assessing this, let us take stock. We have decided that IA is the denial of alternative complementarity, but in no sense appeals to a freeze-

\footnotetext{
7 Note that in speaking of identity here, we are not speaking about who is who at a time. Temkin suggests that IA rules out consideration of identity, on the ground that "if two situations are exactly alike in all other respects, except that they involve different people, then for any factor $f$ relevant to the ranking of outcomes, the two situations must be equally good regarding $f$ " (p. 342, fn. 28). But the real focus of considerations about identity in the present discussion is not that old warhorse, but the thought that considerations of re-identification are ruled out by IA. (I maintain that this is a mistake too, of course.) It is one thing to worry about whether it is the same person again, and another to worry about which person that same person is; the former is what is at issue here.
} 
frame conception of an outcome or situation. We can therefore imagine a role for PAV-style considerations, in which we assess each situation or course of events for the good and harm that is done to individuals in them. Having done all that, we award a score to each situation, in a way that respects the demands of transitivity. We might say, therefore, that PAV itself does not introduce considerations that are essentially comparative, since it can be operated within a purely non-comparative environment. This point is missed, of course, if one works with freeze-frame examples, as Temkin does, following Parfit.

Temkin could however reply to this that the situation he is discussing is only inessentially a freeze-frame one. In the diagram displayed earlier, we could take A and B as diachronic worlds, each with two long-term communities. If so, Temkin would say, PAV itself gives us no reason to prefer either to the other. For Parfit and Temkin interpret PAV as a kind of Pareto principle which says that "one situation is better than another if there is someone for whom it is better and no-one for whom it is worse," (p. 319). And if we interpret it this way, we do get something that involves genuine alternative complementarity. Pareto-interpreted, PAV holds that the sufferings of the worst off in A are only relevant to the comparison of A and B if those people are present in B. It also holds, as Temkin says, that "if the people in A were completely different from those in $\mathrm{B}$, then PAV would yield no reason to prefer A or B" (p. 320).

My own view at this point is that what we have really been shown is how far the Pareto interpretation is from an intuitive understanding of PAV. The suggestion that value derives from the extent to which people are doing better or worse is a long way from the constraint that situations can only be compared to the extent that they contain the same people. That constraint certainly does introduce essentially comparative considerations. But this should not perturb defenders of IA, who should simply reject the Pareto interpretation.

There is anyway more than one Pareto-style way of reading PAV. On what I think is the most natural interpretation, as I have said, PAV concerns changing people's lives for the better or for the worse. We have already seen that this interpretation offers no threat to transitivity. But there are two further interpretations, of Pareto style. On one, PAV announces that the quality of an alternative depends on the quality of the lives there lived. On the other (the one which Temkin adopts) it holds that situations can only be compared to the extent that they contain the same people. I think that the first of these has an equal right to be called a Pareto interpretation, since it is amenable to a certain conception of Pareto-optimality, but it is one which considers lives as slots, and says that the quality of an alternative depends on the quality of the slots occupied in it, irrespective of who is occupying them. As a Pareto view, it holds that one alternative is better than another if in it some better slots are occupied and no worse ones. And, like the first interpretation, it offers no threat to transitivity. Since it is available to proponents of IA, there is nothing in Temkin's discussion of PAV to undermine the prospects of IA and transitivity. It is only by imposing a very peculiar and 
quite optional reading of PAV that Temkin succeeds in showing it to be essentially comparative.

Finally I turn to Temkin's last example:

utility is essentially comparative (UEC): where, roughly, the only way to improve a situation regarding utility is to increase the utility of some of those already living in that situation. (p. 321)

Temkin asserts that UEC, so understood, will lead to intransitivities. This is because, yet again, we are dealing with a person-affecting principle, and we saw in the case of PAV that person-affecting principles generate essentially comparative considerations. But the example he gives is inadequate. He considers a version of the Mere Addition Paradox in which the A people would also exist as A+'s better-off group, and where the same people (including the A people) would exist in both A+ and $\mathrm{B}$. He announces, of this scenario, that:

On UEC A will be better than B, because loss in utility of existing people cannot be made up for merely by adding more people. And B will be better than A+, because loss in some people's utility can be made up for by sufficient increases in the utility of others who already exist. But, on UEC, A is not better than A+, as it would have to be if UEC were transitive. (p. 322)

This is all a mistake, caused by forgetting that we needed to interpret the person-affecting principle PAV in the essentially comparative Pareto way before results of this sort emerged. UEC has received no such interpretation, and it therefore has no tendency to produce this sort of result. Temkin has confused thoughts about people becoming happier or unhappier (presumably in the same, non-freeze-frame situation) with thoughts about comparing only those situations for utility that we find the same people in, happier in one and unhappier in the other. But the impetus of UEC (now, it appears, quite improperly named) is concerned with making people happier or unhappier, or on their becoming happier or unhappier. The only reason to read this in terms of being happier in one situation and unhappier in another, apart from the way in which PAV was changed by its Pareto interpretation, is the sense that thoughts about making people happier are ruled out by IA in virtue of its ban on considerations of routes, origins and identity, i.e. its freeze-frame nature. And we have long ago decided that that was just a mistake.

In fact Temkin's use of UEC seems exactly to miss the point of its original propounder, Jan Narveson. The original idea was that levels of happiness do not matter morally. What matters morally is increases and decreases in people's happiness. And in that case, we cannot achieve a moral comparison of two worlds in terms of the brute levels of happiness they contain, even if the same people are present in both. They can, however, be compared in terms of the rises and falls in happiness experienced by their occupants, whether or not the same people appear in both situations. But such comparison does nothing to undermine transitivity. 
I conclude that Temkin has failed to show that the leading principles he considers are essentially comparative. IA remains an option, and with it we can retain transitivity.

III

I now turn to the way in which Temkin tries to lay out the notion of the essentially comparative. As already reported, he writes:

\begin{abstract}
A principle (or moral ideal) $f$ is essentially comparative if the relevant and significant factors for comparing two alternatives regarding $f$ may vary depending on the alternatives being compared; and, more specifically, $f$ is essentially pairwise comparative if one must directly compare two alternatives in order to determine their relative ranking regarding $f$. (p. 304)
\end{abstract}

Here are some comments on this definition, in no particular order. First, we should distinguish the idea that the value of an object may depend on what we compare to it, from the idea that the value of an object can depend on what the alternatives to it are or were. We can compare anything with anything. But it is tempting to think that mere comparison does not affect value. We need a real situation to do that, one might say, not a thought experiment, and real situations involve real alternatives. So Temkin's constant talk of comparison should be taken with care. It is not to be found to anything like the same degree in Parfit.

I think that such truth as there is in this does not affect the issues with which we have been concerned. There is indeed a distinction between remarks about alternatives and remarks about what happens when we compare one thing with another. Taking the remarks about alternatives first, alternative complementarity properly understood has often been thought to be obviously irrational. But I have found one example which seems to me to be clearly rational. Wilfred Thesiger crossed the Empty Quarter in Arabia by camel before the 1939-45 war, undergoing great trials, difficulties and dangers. He wrote of his exploits: "I would not myself have wished to cross the Empty Quarter in a car. Luckily this was impossible when I did my journeys, for to have done the journey on a camel when I could have done it in a car would have turned the venture into a stunt." 8

What makes this rational, in my view, is the use of the terms "venture" and "stunt." These seem to be, as one might put it, essentially comparative thick evaluative terms. Whether something is a stunt depends on whether one could have done the same thing much more easily another way. So there is an explanation of the rationality of Thesiger's attitude here. And if so, it seems that at least in circumstances of this sort, alternative complentarity is true.

8 Arabian Sands (London: Longmans, 1959), p. 260. 
But that does nothing to show the truth of a similar view about what we compare with what. For we do not need to think that any two things that we compare are alternatives. I might compare the possession of one picture with the possession of another, and these two possessings are alternatives. But I might also just compare the two pictures, asking which to prefer, and the pictures don't seem to be alternatives to each other exactly. To ask which picture is preferable to the other is not to ask which picture it would be better to own or anything else of the sort. This is the truth in the distinction between remarks about alternatives and remarks about mere comparison. At the end of the day, however, this truth doesn't seem to me to cut much ice for present purposes. ${ }^{9}$ There is a difference between preference and choice, but nonetheless preference is relevantly similar to choice. In both cases one is comparing or ranking with respect to each other things that are conceived of as mutually exclusive. It may be that not all preference rankings are rankings of objects conceived of as mutually exclusive, but those that are are ones of which we will say whatever we want to say about the ranking of alternatives.

What this means, of course, is that the idea of a Great Ordering of Values is in trouble. We might hope that we can at least establish a fixed value for each alternative, and thereby place it somewhere in the Great Ordering. But though it is true, in a sense, that that alternative will stay where it is put in the Great Ordering, we cannot appeal to its place in the ordering to answer any new questions about whether it is preferable to some new object, relative to which we have not yet ranked it. Essentially, the Great Ordering can be no more than a record of past evaluations, and cannot serve as a guide in new cases. And if it cannot do that, it is not really much use.

This last point remains good even if one explicitly relativizes one's alternatives to each other, so that what one ranks in the Great Ordering is not the two alternatives $\mathrm{A}$ and $\mathrm{B}$, but the relativized non-alternatives " $\mathrm{A}$ when the alternative is $\mathrm{B}$ " and " $\mathrm{B}$ when the alternative is $\mathrm{A}$." It remains true that the place of these in the Great Orderings can tell us nothing about how to rank the original $\mathrm{A}$ and $\mathrm{B}$ if a third alternative, " $\mathrm{C}$ when the alternatives are A and B," appears. Again, the ranking turns out to be no use at all other than as a record of the past, one that is as gappy as the past is.

My second comment on Temkin's definition above is that he talks of "comparing two alternatives regarding $f$." This awkward phrase does not mean "comparing two alternatives to see which is more $f$ than the other." There is no suggestion that the "relevant and significant factors" may vary for that purpose. It means "comparing two alternatives to see how much their being $f$ affects their value." 10

\footnotetext{
${ }^{9}$ I consider these issues in the final chapter of my Ethics Without Principles.

10 We know this from the glosses given by Temkin immediately after this definition. And if it were not so, there would be no way to save a later claim from falsehood. On p. 305 Temkin considers the Principle of Substitution for Equivalence, which holds that "Given any concept $c$, for all $x, y$ and $z$, to which $c$ is appropriately applied, then regarding
} 
This being so, how are we to understand the talk of "relevant and significant factors"? This heavy phrase recurs in Temkin's paper constantly, but perhaps it means no more than "the things to look at in order to determine how much their being $f$ affects the value of each of two alternatives." The suggestion would then be that one has to look at different things, according to the alternatives one is considering. This may seem too much of a truism to be worth serious attention. But the work to which that Temkin puts it is far from trivial. For in his hands it becomes a crucial part of a proof that if we have any principles or ideals of restricted generality (as in all probability we will have), then even if they themselves are not essentially comparative, our all-things-considered judgements will be.

Consider again the person-affecting view:

PAV: how good or bad an outcome is, is a function of the extent to which it affects people for better or worse.

Temkin considers the suggestion that we try to avoid intransitivity by restricting the scope of PAV "by only allowing it to influence our judgements in cases where exactly the same people are involved." (p. 325) He continues:

suppose ... that A, B and C are three alternatives such that, given its restricted scope, PAV is relevant only in comparing A and C. It could then be the case that all things considered - that is, in terms of all the relevant and significant principles for making each comparison - A is better than B, and B is better than $\mathrm{C}$, yet $\mathrm{C}$ is better than $\mathrm{A}$. After all, even if $\mathrm{C}$ is worse than $\mathrm{A}$ in terms of the principles relevant for comparing $\mathrm{A}$ with $\mathrm{B}$, and $\mathrm{B}$ with $\mathrm{C}$, the extent to which this is so might be outweighed by the extent to which $\mathrm{C}$ is better than A regarding PAV. (ibid.)

The picture we are being offered here has it that there is a certain number of principles relevant for comparisons, some of which are relevant only to some comparisons and some of which are relevant to all. Suppose we have three alternatives, $\mathrm{A}, \mathrm{B}$ and $\mathrm{C}$, and 6 principles, of which the last is only relevant to comparing A and C. We might get the following scores:

$c$, if $x$ is equivalent to $y$, however $x$ compares to $z$ that is how $y$ compares to $z$ " Temkin claims that any essentially comparative concept will break this principle. But if this principle meant, in the case of equality, that if $x$ has more equality than $y$ and $y$ than $z x$ has more than $z$, it surely remains true even if we allow that equality is essentially comparative. It is only if we take the principle to concern how much value $x, y$ and $z$ derive from their respective amounts of equality that it will be broken by any essentially comparative concept. 


$\begin{array}{lllllll}\text { Principles: } & 1 & 2 & 3 & 4 & 5 & 6 \\ \text { A } & 1 & 3 & 2 & 1 & 2 & -2 \\ \text { B } & 2 & 2 & 1 & 2 & -1 & ? \\ \text { C } & 1 & 0 & 0 & 1 & 0 & 10\end{array}$

The idea is that in comparing $\mathrm{A}$ to $\mathrm{B}$ and $\mathrm{B}$ to $\mathrm{C}$ we are only allowed to consider scores under principles 1-5. (This is why there cannot be a score for $\mathrm{B}$ under principle 6.) But when we turn to comparing $\mathrm{A}$ to $\mathrm{C}$, we must also consider scores under principle 6 . This gives us the result that $\mathrm{A}>\mathrm{B}, \mathrm{B}>\mathrm{C}$ and $\mathrm{C}>\mathrm{A}$. If we only considered common criteria, there would be transitivity. But with principles of restricted scope, there will not be.

Now why is it the case that A's score of -2 under principle 6 is not relevant in determining whether $\mathrm{A}$ is all-things-considered better than $\mathrm{B}$ ? Or, to put it another way, why is it that we would be wrong to give $\mathrm{B}$ a score of 0 under principle 6 ? And doing that has a significant advantage we would preserve transitivity.

Let us consider an analogy: the sexual activity principle (SAP): a person's value is a function of their sexual behavior.

Suppose that we are persuaded to restrict this principle so that we only allow it to influence our judgements in cases where the people we are evaluating are married (not necessarily to each other). And suppose that this restricted principle SAP+ is principle 6 on our chart. Suppose further that $\mathrm{A}$ and $\mathrm{C}$ are married persons, and that $\mathrm{C}$ (who is otherwise a fairly indifferent person) gets a truly heroic score for determined fidelity in the face of great temptation, while $A$ has a minus score because of the occasional marital lapse. If we give $\mathrm{B}$, who is not married, 0 in this column, on the grounds that the sexual behavior of the unmarried neither adds to nor subtracts from their overall score, so that the one is not affected by the other, this is surely one way of capturing that fact. And there is surely a rationale for awarding a score of 0 to $B$, which is that this enables one to consider A's sexual behavior as relevant to our overall assessment of $\mathrm{A}$ even when we are comparing $A$ with $B$. In considering whether $A$ is a better person than B overall, we are surely not prevented from counting the effects of A's marital weaknesses merely by the fact that B is not married. What if B especially decided not to get married because she predicted that she would not be faithful? Is she then to be deprived of the gains she will make in comparison with those who do take the plunge and then fall? (Of course she also deprives herself of the benefits of constancy.) Indeed otherwise we will find ourselves with very gappy charts, and end up with the peculiar result that overall comparative evaluation becomes identical with an evaluation that considers only criteria relevant to all the objects evaluated equally. 
What, I think, persuades Temkin that we should put "n/a" or "?" for B's entry under column 6 rather than 0 is the phrase "relevant and significant factors" - especially the word "relevant." The idea is that in pursuit of the thought that we should not allow a principle to influence our judgements in a certain range of cases, we announce that it is not relevant to any comparison involving those cases. This is then interpreted as meaning that scores according to that principle are only marked up in other cases. It is this last move that is suspect. I can surely announce that sexual behavior can do nothing to affect my evaluation of those who are not married, without choosing this way of recording the fact.

There is an ambiguity that is causing problems here:

a relevant principle for comparison is one in terms of which the two objects can properly be compared $v$ s.

a relevant principle for comparison is one which can af-

fect the results of our comparative evaluation.

In announcing that a restricted principle is of the first sort in some cases and not in others, we do nothing to show that in the latter cases the principle is not of the second sort. In our example of sexual activity, I may decide not to consider the sexual activities of one of the two people I am comparing, because she is unmarried. Matters of sexual behavior, I maintain, are not relevant to the evaluation of the unmarried. But they are relevant, in the second sense, to the comparison, since they can affect the overall result by affecting our evaluation of the married person.

I conclude that nothing in the idea of a restricted principle requires us to adopt the sort of essential comparativity that Temkin is trying to foist on us. We are therefore free to adopt his own solution to the Mere Addition Paradox, accepting IA and rejecting intransitivity. Nothing in what Temkin says has proved to be a reason in favor of alternative complementarity. This, of course, does not show that such reasons are not going to be forthcoming. ${ }^{11}$

Jonathan Dancy

The University of Reading and the University of Texas at Austin

Department of Philosophy

j.p.dancy@reading.ac.uk

jdancy@mail.utexas.edu

\footnotetext{
11 I am grateful to an initially anonymous referee (actually Jamie Dreier) for suggestions about how to make this rather complex paper somewhat easier to follow.
} 